\title{
El lenguaje infantil en situaciones de juego en el Jardín de Infantes
}

\section{Children's language at play in Kindergarten}

\author{
María Soledad Manrique ${ }^{1}$ y Celia Renata Rosemberg \\ CIIPME (CONICET) Centro Interdisciplinario de Investigación en Psicología y \\ Matemática Experimental. \\ Universidad de Buenos Aires, Argentina
}

(Recepción: Octubre 2009 - Aceptación: Diciembre 2009)

\begin{abstract}
Resumen
Este trabajo presenta un análisis del lenguaje que emplean los niños de 4 y 5 años de sectores marginados en diferentes tipos de situaciones de juego en el Jardín de Infantes: el juego con instrucciones, el juego en el patio, el socio dramático en rincones y coordinado por la maestra. En particular, se analizan las emisiones infantiles, considerando las funciones para las que emplean el lenguaje (Halliday, 1975) y el grado de explicitud y descontextualización del lenguaje (Chafe, 1985). Los resultados mostraron qu, en todos los tipos de juego, las emisiones infantiles están principalmente destinadas a dar y pedir información y a regular la propia acción y la de los otros. Sólo un porcentaje marginal de las emisiones infantiles presentan formas lingüísticas descontextualizadas. En la mitad de los casos, éstas se producen durante el juego dramático en rincones y sobre todo en interacción con la maestra y no entre pares.

Palabras clave: Lenguaje infantil - funciones del lenguaje - usos descontextualizados de lenguaje - Juego - Jardín de Infantes.
\end{abstract}

\begin{abstract}
The study presents an analysis of the language of children from marginalized urban sectors aged 4 and 5, in different types of play situations at kindergarten: instruction games, games in the playground, sociodramatic play in corners and sociodramatic play coordinated by the teacher. The analysis of children's utterances focuses particularly on preeschoolers' functional uses of languaje (Halliday, 1975) and the production of decontextualized language (Chafe, 1985). Results indicate that children's utterances are mainly destined to give and ask information and to regulate their actions and those of others. Only a small percentage of the total of childlren's utterances present decontextualized language. In half the cases during dramatic play in corners, and mostly when interacting with the teacher, not among peers.
\end{abstract}

Key words: Child language -functional uses of language - decontextualized use of language - play kindergarten.

1 Correspondencia: María Soledad Manrique y Celia Renata Rosemberg. Tte. Gral. Perón 2158. Capital Federal. Argentina. C. P. 1040. Teléfonos: (011) 4745 - 3953 - (011) 4542 - 2214. E-mail: solemanrique@yahoo.com.ar crrosem@hotmail com 


\section{Introducción}

El estrecho vínculo entre el juego y el desarrollo infantil ha constituido un foco importante de reflexión y de conceptualización. Tanto Piaget (1962), como Vygotski (1988), Bruner (1986) y Nelson (1996) han destacado la función del juego como potenciador del desarrollo infantil. El juego contribuye al dominio de las situaciones porque el niño las elabora a partir de la propia acción, y, por tanto, disminuye la ansiedad ligada a las mismas (Rivière, 2003). El juego refleja ese dominio del entorno y a su vez, depende de él para su desarrollo. El rasgo fundamental que lo caracteriza es su naturaleza simbólica: al igual que el lenguaje, implica la evocación de significados ausentes por medio de significantes diferenciados (Piaget, 1962).

Lenguaje y juego mantienen una relación bidireccional, de influencia mutua: por un lado el lenguaje permite desarrollar la trama del juego y sostenerlo en el tiempo y, por otro lado, el juego, al activar esquemas de conocimiento compartido, facilita el empleo por parte del niño de recursos lingüísticos más complejos (Nelson, 1996, Rosemberg, 2008; Manrique, 2009).

En sus investigaciones cuasi experimentales sobre juego y lenguaje infantil Pellegrini (1982, 1984, 1985, 1985b, 1986) se focalizó en las funciones del lenguaje empleado por los niños y en el uso de lenguaje descontextualizado. En sus trabajos centrados en las funciones del lenguaje, codificó las emisiones de los niños durante distintos tipos de juego - sociodramático, de construcción y de arte - siguiendo el modelo propuesto por Halliday (1975). De acuerdo a este autor, los niños en edad preescolar emplean el lenguaje con una serie de funciones - representacional, interpersonal, personal, regulatoria, heurística, instrumental, e imaginativa -. Con el desarrollo, cada emisión infantil tiende a cumplir más de una función. Pellegrini (1984) observó que en el juego sociodramático los niños empleaban el lenguaje para cumplir con una mayor variedad de funciones que en otros tipos de juego.

En otros de sus trabajos Pellegrini (1982, 1985, 1986) atendió al uso por parte de los niños de lenguaje descontextualizado para crear una situación lúdica. Esta forma de lenguaje se caracteriza por su explicitud y su independencia del contexto de situación, tanto durante el proceso de producción de significados como en la interpretación (Chafe, 1985). Para ello se vale de un léxico preciso y de una serie de recursos lingüísticos elaborados, tales como la subordinación, la nominalización y el establecimiento de referencias endofóricas. Esta formas de lenguaje propias de la escritura pueden también ser utilizada en la oralidad. En este sentido, Pellegrini mostró que las formas descontextualizadas (Chafe, 1985) eran empleadas sobre todo en el juego socio-dramático y en mayor medida por los niños de 5 años que por los de 3. Pellegrini sostiene que esto se debe a que en este tipo de juego los niños comparten con otros la situación imaginaria que van construyendo y las transformaciones de los objetos y de su propio rol. Para ello deben recurrir a formas lingüísticas en las que priman las referencias endofóricas -los elementos se nombran, se definen y caracterizan dentro del texto y no por referencias al contexto de situación.

Investigaciones más recientes realizadas con niños brasileros de 16 a 28 meses (Hauer, Altamura \& Anisfeld, 2007) han corroborado esta relación entre el juego sociodramático y el desarrollo lingüístico de los niños, medido de acuerdo a la versión Brasilera del Inventario MacArthur, que considera la amplitud del vocabulario, la capacidad combinatoria, MLU (largo promedio de la emisión), y el empleo de sufijos morfológicos.

Cuando el foco de interés es el lenguaje que los niños emplean en contextos naturales en el Jardín de Infantes, y no en contextos creados cuasi experimentalmente, como los estudiados por Pellegrini, otras variables, además del tipo de juego y de la edad de los niños pueden afectar las formas del lenguaje infantil.

En este sentido, diversas investigaciones se han ocupado de las formas que adopta la mediación adulta en el juego infantil. Así por ejemplo, los trabajos de Bruce (1992), Enz y Christie (1997), Bondioli (2001), Sarlé (2006) y Morrow \& Schickedanz (2006) han mostrado que la participación de los maestros en el juego implica un continuo: en un extremo el docente sólo contempla el juego 
de los niños y en el otro, interviene tanto que el juego se diluye. Bruce (1992) ha identificado estas formas de mediatización adulta con el nombre de: “dejar hacer”, “didáctico” e "interaccionista”. Los trabajos de Tamis Le Monda, Bornstein, Baumwell \& Damast (1996), y los de Rosemberg (2008), mostraron, asimismo, que ciertas formas de participación adulta en el juego contribuyen a que éste sea más extenso y complejo. Se trata de la forma de participación "interaccionista": el docente proporciona nuevos contenidos y enriquece el juego de los niños, participando contingentemente de él.

La investigación de Sarlé (2006), describió las diferencias en las formas de mediación de las maestras en función del tipo de juego - dramático, con objetos y con reglas convencionales. En el juego dramático, la maestra puede enriquecer la propuesta ampliando la base de experiencia que nutre las situaciones imaginarias, movilizando el contenido del juego en función del guión que los niños van construyendo. En cambio, en el juego con objetos el rol de la maestra se ve influido por el tipo de objeto involucrado en el juego (Enz y Christie, 1997) y por la consigna del juego. Por último, los juegos de reglas requieren que el docente enseñe las reglas hasta que las mismas sean aprendidas (Garrido, 2008).

Sarlé (2006), en su análisis de los contextos de juego en Jardines de Infantes en Argentina y Chile, encontró que las formas de mediación de las maestras también estaban condicionadas por la estructura didáctica del juego, esto es, por el modo en que se organiza el grupo y los espacios, la cantidad de niños por tarea, la posibilidad de los niños de elegir a qué jugar y el grado de visibilidad del desempeño de cada niño. Pero, ni en los trabajos de Sarlé ni en los otros trabajos citados se ha considerado cómo la estructura didáctica y las intervenciones docentes en las situaciones de juego, no pueden incidir en el uso del lenguaje infantil es estas situaciones.

En esta línea, el presente trabajo tiene precisamente por objeto analizar el lenguaje infantil en situaciones de juego en el Jardín de Infantes. Se atiende tanto a las funciones (Halliday, 1975) predominantes que cumple el lenguaje en el juego, como al empleo de formas de lenguaje descontextualizadas (Chafe, 1985). Se consideran variaciones en estos aspectos según el tipo de juego, el momento del juego y la intervención de la maestra en él.

\section{Método}

\section{Recolección de información empírica}

Este trabajo es parte de una investigación que tuvo por objeto contribuir al conocimiento de los procesos de interacción lingüística que tienen lugar en Jardines de Infantes de poblaciones urbano - marginales, en tanto estos procesos de interacción constituyen un contexto para el desempeño y el desarrollo lingüístico y cognitivo de los niños de 3 a 5 años. Con ese fin se consideraron distintos tipos de actividades en que participan frecuentemente los niños en el Jardín de Infantes, las estrategias de interacción de las maestras, así como la relación entre estos dos aspectos y el desempeño lingüístico - discursivo y cognitivo de los niños (Manrique, 2009).

En este marco, el corpus del trabajo está conformado por 25 situaciones de juego, que tuvieron lugar en el curso de tres jornadas completas de clase de 8 grupos de niños (127 niños) de 4 y 5 años pertenecientes a dos centros infantiles que atienden a poblaciones urbano - marginadas en la provincia de Buenos Aires, Argentina - 22.40 horas de filmación.

La información se recolectó por medio de registros en audio y video transcriptos en detalle.

Con el fin de comparar las situaciones de juego, estas fueron agrupadas en cuatro tipos, según sus características: juego dramático grupal, juego con reglas convencionales, juego dramático en rincones y juegos de destreza física en el patio.

Los juegos dramáticos grupales son actividades en las que las maestras se valen de un guión narrativo para representar un mundo imaginario en el que intentan incorporar a los niños en el curso 
del juego. Estos participan respondiendo o no a la propuesta de la maestra, o expandiéndola pero es la maestra quien planifica, guía y desarrolla el juego.

Los juegos con instrucciones tienen por objetivo superar a otros participantes, respetando determinadas reglas. Los juegos observados fueron llevados a cabo por el grupo total junto con la maestra.

El juego en rincones o sectores, habitual en los Jardines de Infantes, consiste en el juego paralelo de diferentes grupos de niños en diferentes sectores del aula, elegidos por los niños. En los Jardines de Infantes en los que se realizó la investigación este juego contaba con dos rincones básicos: el de los autos y el de la casita. En ambos rincones los niños llevaban a cabo el juego simbólico o socio - dramático, actualizando su conocimiento de eventos y de la estructura narrativa para recrear situaciones imaginarias.

En el patio durante el recreo se observaron juegos motores, que involucran destreza y despliegue físico y menos comunicación lingüística, tales como el fútbol, carreras corporales o con vehículos de juguete y rondas.

El corpus comprende 8 situaciones de juego dramático grupal, 4 de juegos con instrucciones, 9 de juego dramático en rincones y 4 de juego en el patio.

\section{Procedimiento para el análisis de la Información}

Con el propósito de caracterizar el lenguaje empleado por los niños durante el juego, se segmentaron las situaciones en tres momentos recurrentes en la mayoría de los casos: antes durante y después del juego. El momento previo al juego y el de cierre no formaron parte de todas las situaciones registradas.

Se recurrió a un procedimiento cuantitativo de análisis atendiendo a categorías elaboradas en investigaciones antecedentes que resultan pertinentes. Las variables dependientes consideradas fueron las siguientes:

1) Funciones para las cuales los niños empleaban el lenguaje: Para determinar la frecuencia con que se empleaba el lenguaje con diferentes funciones en el total de situaciones y según el tipo de juego y el momento del mismo, se consideraron las categorías elaboradas por Halliday (1975). Si bien cada emisión puede cumplir con varias de estas funciones simultáneamente, en el presente trabajo se categorizó cada una de las emisiones infantiles de acuerdo a la función predominante:

a) uso informativo: proporcionar o elicitar información.

KEVIN: Yo jugué con los animales de la granja./ TOMÁS: ¿A qué juegan?

b) uso instrumental: organizar una tarea u obtener resultados.

AIXA: Llevá esta silla. /AGOSTINA: La ropa de la bebé, la percha de la ropa (le entrega una percha).

c) uso regulativo: regular las conductas de los otros o la propia.

EVELYN: Vení Joaquín. /TATIANA: Atrás mío, usted atrás, no atrás de Rodrigo (Tatiana pone en fila a Evelin, a Rodrigo y a Joaquín) Bueno vos adelante, ahí, después vos, dale....como hago yo.-.

d) uso imaginativo: crear mundos imaginarios.

SERGIO (A KEVIN): Hermano, no, no te tires, aquí hay agua y te vas a ahogar. (Hace que su auto ataje al de KEVIN).

e) uso heurístico: reflexionar sobre la realidad.

CAROLINA (se acerca corriendo y se pone la mano en el pecho): Si te quedas quieta se te mueve el corazón.-.

f) uso personal: expresar o elicitar la expresión de sentimientos. 
JOAQUÏN: No quiero jugar más, es muy aburrido.

g) uso interpersonal: establecer y mantener la relación con otros.

MARTÍN: Yo no juego con vos. Me vas a molestar.

2) El uso de formas de lenguaje descontextualizado (Chafe, 1985)

Elián, Kevin, Sergio y Ludmilla juegan con autitos en uno de los rincones.

KEVIN: La policía, viene la policía, dale.

MAESTRA: ¿A qué juegan ustedes?

ELIAN: Jugamos a que Kevin y Sergio son los policías y yo era ladrón.

KEVIN: Y nos quería atrapar.

LUDMILLA: Había un señor.

SERGIO: No, era un policía. (Pone el auto sobre el estacionamiento y choca al auto de Ludmilla) Mirá, Ludmilla es policía.

KEVIN: No, los policías no se "pelian”. Los policías arrestan a los que se portan mal. Yo soy policía.

Se tomaron como indicadores de estilo de lenguaje descontextualizado el léxico preciso -policía / ladrón - y una serie de recursos lingüísticos elaborados, tales como la subordinación - Jugamos a que Kevin y Sergio son los policías, la nominalización y el establecimiento de referencias endofóricas.

Las funciones para las cuales los niños empleaban el lenguaje así como el uso de formas lingüísticas descontextualizadas se analizaron atendiendo a los tipos y momentos del juego así como también a si la interacción se realizaba entre pares o con la maestra.

\section{Resultados}

El análisis del lenguaje infantil en las situaciones de juego en el Jardín de Infantes mostró que el lenguaje empleado por los niños, tanto en lo que se refiere a las funciones empleadas, como en lo que atañe a las formas descontextualizadas que adopta, varía dependiendo del tipo de juego, del momento del juego y de la intervención docente en él. En las tablas 1 a 5 se presentan los resultados del análisis cuantitativo.

En el la tabla 1 se presentan los porcentajes de participación de la maestra y de los niños en las situaciones de juego completas y distinguiendo los momentos que comprenden las situaciones.

Tabla 1: Porcentaje de turnos de intervención de la maestra y de los niños en la situación completa y según los momentos de la misma.

\begin{tabular}{|l|c|c|c|c|}
\hline Tipo de juego & Dramático grupal & Reglas & $\begin{array}{c}\text { Dramático } \\
\text { en rincones }\end{array}$ & Motor en el Patio \\
\hline MArticipante & $52,57 \%$ & $55,34 \%$ & $48,83 \%$ & $40,66 \%$ \\
\hline NIÑOS & $47,43 \%$ & $44,66 \%$ & $51,17 \%$ & $59,34 \%$ \\
\hline TOTAL & $\begin{array}{c}100 \% \\
(1267)\end{array}$ & $\begin{array}{c}100 \% \\
(721)\end{array}$ & $\begin{array}{c}100 \% \\
(1583)\end{array}$ & $\begin{array}{c}100 \% \\
(241)\end{array}$ \\
\hline
\end{tabular}


Como se observa en la tabla, si no se discriminan los diferentes momentos de las situaciones, la maestra y los niños participan en una proporción similar (M: 50.79\% vs. N: $49.21 \%$ ) durante las situaciones de juego. La mayor parte de las emisiones de las maestras y de los niños tienen lugar durante el juego (1261 - 1313). Este es el momento más largo (2574), seguido por el momento anterior al juego (666), mientras que el momento en que menos intercambios se registraron fue después del juego (572).

Si se considera la participación de las maestras y de los niños en los diferentes momentos de las situaciones de juego, se observa que antes del juego, se incrementa la diferencia a favor de la maestra (M: 59\% vs. N: 40.99\%). Esta diferencia en la participación es estadísticamente significativa al 5\%, usando el Test de Hipótesis para dos proporciones con un p valor de 0.0107 según la prueba exacta de Fisher.

El intercambio que se produce de modo previo al juego constituye el único momento de la situación en el que hay una diferencia relativamente importante a favor de uno de los participantes, la maestra. Esto probablemente se deba a que en el momento previo al juego las maestras establecen el marco de la actividad. Si bien en algunas situaciones este marco está implícito porque al constituir una rutina del grupo no requiere ser explicitado, en otros casos da lugar a un intercambio extendido en el que se explica o planifica el juego. Las emisiones de los niños en este momento del juego tienen lugar como respuesta a las intervenciones de algunas de las maestras que proporcionan las consignas a través de preguntas que los niños deben responder. En lugar de explicar ellas mismas las reglas, o los permisos y licencias del juego, muchas maestras lo elicitan de sus alumnos. Como se trata de conocimiento compartido los niños pueden contribuir al intercambio poniendo en común este conocimiento y acordando en conjunto este marco.

En la tabla 2 se presentan los datos referidos a la participación relativa de las maestras y de los niños en los diferentes tipos de juego.

Tabla 2: Porcentaje de los turnos de intervención de la maestra y de los niños en los diferentes tipos de juego.

\begin{tabular}{|l|c|c|c|c|}
\hline & TOTAL & ANTES & DURANTE & DESPUÉS \\
\hline \multirow{2}{*}{ MAESTRA } & $50,79 \%$ & $59 \%$ & $48,99 \%$ & $49,30 \%$ \\
& $(1936)$ & $(393)$ & $(1261)$ & $(282)$ \\
\hline \multirow{2}{*}{ NIÑOS } & $49,21 \%$ & $40,99 \%$ & $51,01 \%$ & $50.69 \%$ \\
& $(1876)$ & $(273)$ & $(1313)$ & $(290)$ \\
\hline \multirow{2}{*}{ Total } & $100 \%$ & $100 \%$ & $100 \%$ & $100 \%$ \\
& $(3812)$ & $(666)$ & $(2574)$ & $(572)$ \\
\hline
\end{tabular}

Como se observa en la tabla 2 los porcentajes de turnos de intervención de las maestras y de los niños varían en las diferentes situaciones. Cuando los niños eligen a qué jugar, como en el patio o en rincones, es mayor el porcentaje de sus intervenciones en relación a las de la maestra (patio: N: 59.34\% vs. M: 40.66\%; rincones: N: 51.17\% vs, M: 48.83\%). Mientras que en la situación de juego dramático en rincones la diferencia entre las intervenciones de los niños y las de la maestra es mínima (2.34 puntos), en la situación de patio se trata de una diferencia estadísticamente significativa al 5\%, usando el Test de Hipótesis para dos proporciones con un p valor de 0.0160 según la prueba exacta de Fisher.

En las situaciones en las que la maestra es quien propone el juego, su porcentaje de turnos es mayor que el de los niños, aunque la diferencia no resulta estadísticamente significativa. Este resultado es el esperado dado que la maestra es quien coordina y guía el juego en su totalidad.

Los resultados del análisis de las funciones para las que los niños emplean el lenguaje según el tipo de juego y los distintos momentos del juego, se presentan en la tabla 3. 


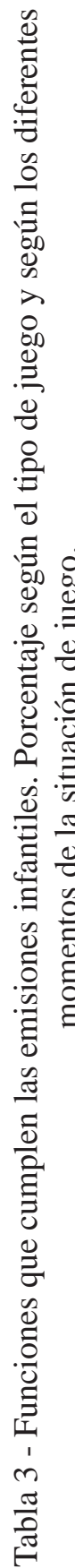

\begin{tabular}{|c|c|c|c|c|c|c|c|c|}
\hline \multirow{4}{*}{ 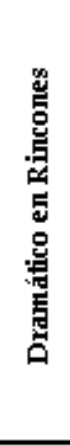 } & 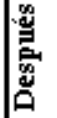 & 恕 & $\begin{array}{l}2 \\
\vdots \\
0 \\
0\end{array}$ & 30 & 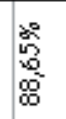 & $\stackrel{2}{2}$ & 点 & 总 \\
\hline & 蒙 & స్టి & 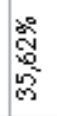 & 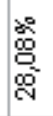 & 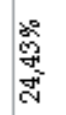 & 今ึ & 商 & 总 \\
\hline & 产 & 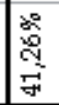 & 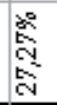 & 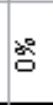 & 胥 & 㺼 & 点 & 总 \\
\hline & 要 & 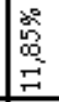 & 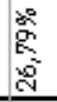 & $\begin{array}{l}3 \\
0 \\
2 \\
2 \\
2 \\
-1\end{array}$ & $\begin{array}{l}3 \\
2 \\
2\end{array}$ & 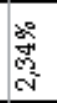 & 胥 & 总 \\
\hline \multirow{4}{*}{ 兽 } & 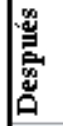 & 80 & 8 & వ̊ & మ̊ & 8 & b̊ & 总 \\
\hline & 鸏 & 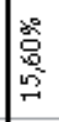 & 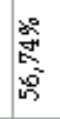 & $\begin{array}{l}\infty \\
\infty \\
0 \\
\sim \\
\sim\end{array}$ & 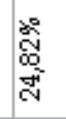 & E & مू & 总 \\
\hline & 旁 & 80 & 8 & 3 & s。 & 3 & 30 & 总 \\
\hline & 兽 & 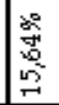 & 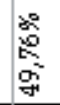 & స્ㅇ & $\begin{array}{l}\stackrel{2}{2} \\
\stackrel{2}{0}\end{array}$ & 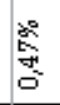 & 点 & 总 \\
\hline \multirow{4}{*}{ 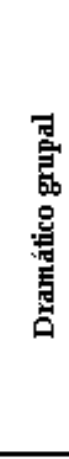 } & 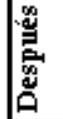 & 驾 & 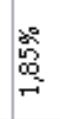 & 今̊ & 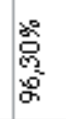 & 恣 & مू & 谷 \\
\hline & 蒙 & 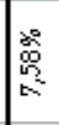 & 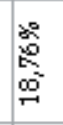 & F & 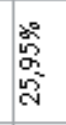 & $\begin{array}{l}\stackrel{8}{2} \\
\text { 心 }\end{array}$ & $\stackrel{2}{2}$ & 莕 \\
\hline & 章 & 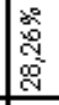 & 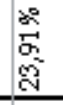 & 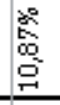 & 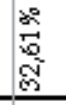 & $\begin{array}{l}2 \\
2 \\
2 \\
2 \\
7 \\
7\end{array}$ & 3 & 总 \\
\hline & 焉 & 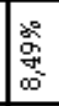 & 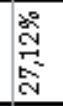 & 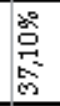 & $\begin{array}{l}\text { 炙 } \\
\text { 怘 } \\
\text { 品 }\end{array}$ & 心్ & $\begin{array}{l}\infty \\
0 \\
0 \\
\infty \\
\infty\end{array}$ & 总 \\
\hline \multirow{4}{*}{ 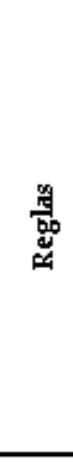 } & 誩 & $\mathrm{b}^{\circ}$ & 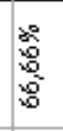 & 3 & 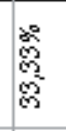 & 今̊ & 3 & 菅 \\
\hline & 蒙 & 芯 & 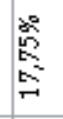 & 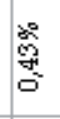 & 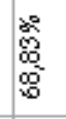 & 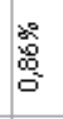 & bे & 总 \\
\hline & 竞 & 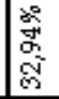 & 总 & 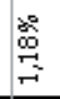 & $\begin{array}{l}0 \\
0 \\
0 \\
0 \\
0 \\
5 \\
5\end{array}$ & \$̊ & 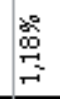 & 总 \\
\hline & 预 & 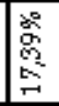 & 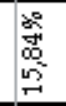 & 莺 & 谞 & 总 & $\frac{2}{5}$ & 总 \\
\hline & 总 & 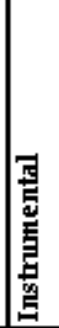 & 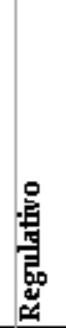 & 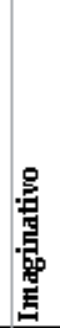 & 兽 & 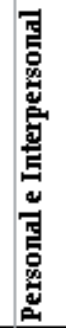 & 总 & $\begin{array}{l}\text { 是 } \\
\stackrel{0}{\circ}\end{array}$ \\
\hline
\end{tabular}


Si se atiende en la tabla a los usos del lenguaje sin discriminar ni tipo ni momento del juego, se observan diferencias importantes en la función que cumplen de modo preponderante las emisiones infantiles. Estas se encuentran principalmente destinadas a dar y pedir información (42.64\%). En segundo término, tienen como función regular su acción y la de los otros (24.25\%) con el objeto de coordinar su juego con el de los demás. En tercer término se registran las emisiones de los niños que están destinadas a crear mundos imaginarios en el marco del juego dramático (18,12\%).

El relativamente bajo porcentaje de emisiones destinadas a crear mundos imaginarios pone de manifiesto el hecho de que en el juego dramático, los niños no siempre recurren al lenguaje, sino que suelen realizar acciones que imitan quehaceres de personajes de películas o series de televisión o recrean acciones de los adultos en las situaciones de la vida cotidiana. Los niños dramatizan las acciones ellos mismos o utilizan muñecos y se valen sobre todo de onomatopeyas y gestos. En otros casos, en cambio, el uso del lenguaje con función imaginativa les permite a los niños dar forma al papel que se encuentran representando en el juego dramático: las palabras acompañan las acciones que van realizando y explicitan al resto de los participantes lo que están haciendo. Por medio del lenguaje los niños van desarrollando la trama narrativa de su juego. Las formas más socializadas de juego dramático que implican compartir el juego con otros niños, demandan a cada jugador que explicite su rol y ponga en palabras la trama. A partir de esta explicitación, los otros niños pueden integrarse a la situación. Al coordinar las acciones de varios participantes el juego se complejiza, porque estos deben ponerse de acuerdo con respecto a los roles y a la trama.

Los niños también se valen del lenguaje con una función instrumental en el 12,53\% de sus emisiones, para distribuir los objetos que emplearán. El porcentaje de emisiones que tienen una función heurística es muy bajo (1.75\%) y se concentra en los intercambios que se producen durante el juego. Aún cuando en la mayor parte de las emisiones infantiles pueden inferirse las emociones e intenciones de los niños, sólo un pequeño porcentaje de sus intervenciones están exclusivamente destinadas a expresarlas $(1,23 \%)$.

Las diferencias entre cada uno de los valores referidos a las funciones con las que se emplea el lenguaje en el juego son estadísticamente significativas al 1\% aplicando Test de Hipótesis para dos proporciones (p valor de 0.0068).

Si se consideran las diferencias según el tipo de juego, se observa que en el juego con instrucciones las emisiones infantiles tienen por objeto principalmente dar y pedir información (65.22\%), en menor proporción tienen fines instrumentales (17.39\%) y regulativos (15.84\%).

En el juego dramático grupal, el 38.48\% de las emisiones infantiles tienen por objeto informar; el 37.10\% crear mundos imaginarios y el 27.12\% regular la acción. El hecho de que no existan prácticamente diferencias entre la función imaginativa y la función informativa puede responder a las características de este tipo de juego: es la maestra quien plantea la situación imaginaria e insta a los niños a que participen de ella. Si se atiende a las emisiones de los niños en cada uno de los momentos, se observa que durante la situación de juego se incrementa el porcentaje de emisiones infantiles destinadas a crear mundos imaginarios a través del lenguaje (41.11\%). En los intercambios que se producen antes de la situación de juego también un $10.87 \%$ de las emisiones están destinadas a crear mundos imaginarios. La diferencia entre el porcentaje de uso de lenguaje con función imaginativa durante el juego y antes del juego es significativa al 1\% aplicando Test de Hipótesis para dos proporciones con un p valor de 0.0000 según la prueba exacta de Fisher.

En el juego en el patio, la mitad de las emisiones infantiles están destinadas a regular la propia acción o la de los otros (49.76\%) y a informar, un $24.82 \%$, con una presencia casi nula de las otras funciones. De modo que, al igual que el juego con instrucciones, el juego en el patio, es el que demanda a los niños el empleo de un lenguaje menos rico y diverso desde el punto de vista funcional.

En el juego dramático en rincones, los niños usan el lenguaje con fines informativos (43.70\% de sus emisiones), en un $26.79 \%$ para regular las acciones y sólo en un $15.18 \%$ para generar mundos imaginarios a través del lenguaje. Estas emisiones con fines imaginativos se concentran durante el juego, y, en ese momento, representan el $28.08 \%$ de las emisiones. 
Para analizar la complejidad del lenguaje empleado por los niños en el juego se atendió al tipo de juego, al momento de la situación y al participante con quien el niño se encontraba interactuando, suponiendo que estos aspectos podían imponer diferentes demandas lingüísticas a los niños y podían elicitar formas lingüísticas de diferente grado de explicitud y de descontextualización. En la tabla 4 se muestra la distribución de las emisiones infantiles que presentan un estilo de lenguaje descontextualizado en las diferentes tipos de juego y en los diferentes momentos.

Tabla 4: Empleo de formas explícitas de lenguaje por parte de los niños según el tipo de juego y según el momento en el que tiene lugar

\begin{tabular}{|c|c|c|c|c|}
\hline Tipo de juego & Dramático grupal & Reglas & $\begin{array}{c}\text { Dramático } \\
\text { en Rincones }\end{array}$ & Motor en el Patio \\
\hline Antes & $0 \%$ & $54,54 \%$ & $5,55 \%$ & $0 \%$ \\
\hline Durante & $77,77 \%$ & $45,45 \%$ & $53,70 \%$ & $100 \%$ \\
\hline Después & $22,22 \%$ & $0 \%$ & $40,74 \%$ & $0 \%$ \\
\hline Situación Completa & $18 \%$ & $11 \%$ & $54 \%$ & $17 \%$ \\
\hline
\end{tabular}

$100 \%=100$, que equivale al 5,33\% del total del habla infantil.

Sólo el 5,33\% del total de las emisiones infantiles presentan formas lingüísticas descontextualizadas. De este porcentaje de emisiones, la mitad (el 54\%) de las emisiones infantiles tienen lugar durante el juego dramático en rincones. Cuando se distinguen las emisiones según el momento de la situación en el que son producidas, se observa que éstas tienen lugar sobre todo durante el juego (53.70\%) y después del juego (40.74\%). La asociación entre las variables uso descontextualizado del lenguaje y tipo de juego resultó significativa $(\mathrm{p}<0.1 \%)$ medida por medio de la prueba $\mathrm{X}^{2}$.

El empleo de formas de discurso descontextualizado en las situaciones de juego dramático puede explicarse por las demandas que conllevan este tipo de situaciones para los niños. Para crear lazos entre las acciones propias y las ajenas, los niños se valen de recursos lingüísticos que permiten relacionar proposiciones, tales como los subordinantes - "Los policías arrestan a los que se portan mal." Por otro lado, para otorgar una entidad particular a un objeto o personaje en el juego, los niños deben explícitamente lexicalizarlos - "SERGIO: Uiuiuiuiu. [...] Ellos son los policías. El era policía y yo era ladrón. [...]" El uso de recursos como los citados -subordinantes y lexicalizaciones- crea condiciones que favorecen el uso de lenguaje descontextualizado tanto para producir como para interpretar los significados.

Los niños también recurren a formas de lenguaje relativamente descontextualizadas en el transcurso del juego, cuando la maestra, que no ha estado presente, recorre los rincones y pregunta a qué juegan. Estos intercambios entre la maestra y los niños dan lugar a que los niños empleen formas más complejas de lenguaje. Así por ejemplo, ante la pregunta de la maestra“ ¿Por qué los perseguías, qué habían hecho?”-el niño responde empleando un conectivo causal -“Porque yo., rompí... eh ... una cosa que abren... y ante la nueva intervención de la maestra $-¿$ Rompiste una barrera?, el niño responde sin recurrir a deícticos. -“Si, una barrera y Elián rompió el ascensor.” -. El hecho de que la maestra no ha estado presente durante el juego implica que el niño debe valerse de un léxico preciso, con el fin de hacerse comprender - barrera/ asensor-. 
El empleo de formas explícitas de lenguaje se registra en menor proporción en la situación de juego dramático grupal (18\%). Cuando se atiende a los distintos momentos que configuran la situación, se observa que estas emisiones tienen principalmente lugar durante el juego $(77.77 \%$, de las emisiones que presentan un estilo de lenguaje descontextualizado tienen lugar en este momento de la situación). En el transcurso del juego dramático grupal los niños y la maestra van planificando y creando la trama del juego, para lo cual deben emplear formas de lenguaje complejas, - "MAESTRA: ¿Para qué querés darle el remedio?/ TOMAS: Para...porque... para que se le pase.”-.

El 17\% de las emisiones en las que se emplean formas relativamente descontextualizadas de lenguaje se registraron durante el juego en el patio, en general para regular la conducta propia y ajena. En efecto, los niños necesitan valerse de discurso descontextualizado para argumentar en situaciones de conflicto y defender su punto de vista - "JORGE: Seño, él no nos deja jugar con los camiones que trajo de la sala.”-. De hecho el niño recurre a una cláusula relativa con el objeto de explicar la situación a la maestra, que no ha estado presente y buscar su apoyo.

El 11\% de empleo de formas explícitas de lenguaje por parte de los niños durante los juegos con reglas se distribuye en los dos primeros momentos: antes y durante el juego. En el primer momento se emplea en los casos en los que la maestra solicita a los niños que expliquen las reglas del juego -"MAESTRA: ¿Qué había que hacer? A ver quién se acuerda cómo se jugaba. N: Hay que, cuando hay uno, hay que agarrar un corazón.”-

El porcentaje bajo de formas explícitas de lenguaje en este tipo de juego puede explicarse por la referencia a las piezas del juego que se hallan presentes en el contexto de la situación y por el objetivo mismo del juego, que favorece el uso de deícticos - LUANA: ¿Vos tenés esto? (mostrando la pieza que sacó)/ JUAN: Yo./ N: Ahora le toca a él. (Señala a Lucas)/ LUCAS: A mí.”-. El lenguaje contextualizado, propio de un estilo de lenguaje oral, que emplean los niños en este tipo de situaciones es funcional a los requisitos que el tipo de juego plantea. De hecho, los niños se comprenden entre sí y se desempeñan con eficacia cumpliendo con los objetivos del juego.

Los resultados del análisis cuantitativo en el que se considera con quién interactúa el niño (con la maestra o con otro niño), cuando emplea formas descontextualizadas de lenguaje, se encuentran resumidos en la tabla 5, que se presenta a continuación.

Tabla 5: Empleo de formas explícitas de lenguaje por parte de los niños según el participante con el que interactúa.

\begin{tabular}{|l|c|c|c|c|}
\hline Marticipante & Total & Antes & Durante & Después \\
\hline Con niño & $39 \%$ & $22,22 \%$ & $56,92 \%$ & $0 \%$ \\
\hline Con maestra & $61 \%$ & $77,77 \%$ & $43,07 \%$ & $100 \%$ \\
\hline Total & $\begin{array}{c}100 \% \\
(100)\end{array}$ & $\begin{array}{c}9 \% \\
(9)\end{array}$ & $\begin{array}{c}65 \% \\
(65)\end{array}$ & $\begin{array}{c}26 \% \\
(26)\end{array}$ \\
\hline
\end{tabular}

La mayor proporción de emisiones de los niños en las que emplean formas descontextualizadas de lenguaje tiene lugar cuando interactúan con la maestra (61\% vs. 39\%). Esta diferencia es estadísticamente significativa al $1 \%$ (p valor 0,0029). Ahora bien, cuando se consideran los momentos del juego, se observa que el empleo de formas descontextualizadas de lenguaje en interacción con la maestra predomina antes ( $77.77 \%$ vs. $22.22 \%$ ) y después del juego (100\% vs. $0 \%$ ), es decir cuando 
el juego se planifica o cuando se lo relata a otros, pero no durante el mismo. En este sentido cabe tener en cuenta la estructura didáctica en la que los juegos están insertos. Tanto en el momento previo al juego como en el momento posterior, no se observan intercambios entre los niños, sino que la comunicación esta centrada en la maestra, quien organiza el juego, en el momento anterior y elicita la reconstrucción del juego en el momento posterior.

Con respecto al momento previo al juego se observaron dos tipos de situaciones que dan lugar a que los niños empleen formas de lenguaje descontextualizadas, que caracterizan al lenguaje alfabetizado: o bien cuando la maestra solicita a los niños que proporcionen las reglas de un juego o bien cuando solicitan que planifiquen el juego dramático en las situaciones de rincones.

En el momento posterior al juego, en algunas de las situaciones de juego en rincones los niños deben informar acerca de su juego a la maestra y a los otros niños. De ahí que para poder ser comprendidos, deban emplear un lenguaje preciso y estructuras sintácticas más complejas.

El empleo de estas formas de lenguaje más descontextualizadas en el juego en rincones parece responder a las características de este tipo de situaciones. Cuando el adulto solicita al niño que relate su juego ya sea de modo retrospectivo, esto es, el juego que ya tuvo lugar, ya sea de modo prospectivo - es decir, la planificación de su juego, genera una determinada demanda: los niños deben referir a aspectos lejanos en el tiempo y en el espacio. Al responder a esta demanda los niños ponen en juego usos más descontextualizados de lenguaje cercanos al estilo de lenguaje escrito.

Durante el juego, las emisiones de los niños en las que emplean formas de lenguaje descontextualizado son más frecuentes en la interacción con otros niños (56,92\%) que con la maestra (43,07\%), aunque no se trata de una diferencia significativa. Esto puede comprenderse nuevamente si se atiende a la estructura didáctica: en dos de los cuatro tipos de juegos analizados -el juego dramático en rincones y el juego en el patio-, los niños jugaban solos y las maestras participaban ocasionalmente. Si bien muchos niños directamente no recurren al lenguaje en su juego simbólico o sólo emplean frases simples u onomatopeyas, otros pueden valerse de formas descontextualizadas de lenguaje tanto cuando interactúan con otros compañeros, como cuando lo hacen con la maestra. Pareciera se que el empleo de un tipo de lenguaje más complejo, está relacionado con las demandas propias de cada situación interactiva. Cuando los niños necesitan referirse a aspectos lejanos en el espacio o en el tiempo, como ocurre cuando planifican su juego o explican sus reglas o cuando informan a otros acerca de su juego, emplean en mayor medida formas descontextualizadas de lenguaje. El juego dramático, por el modo en que está estructurado (porque se planifica y se reconstruye al finalizar), es aquél que concentra en mayor medida un uso descontextualizado de lenguaje.

\section{Discusión}

El análisis de las intervenciones infantiles durante las situaciones de juego mostró que los usos más frecuentes del lenguaje por parte de los niños son informar y regular la acción propia y ajena. El hecho de que la función regulativa del lenguaje sea una de las predominantes en las situaciones de juego en general implica que podría extenderse la afirmación de Vygotski (1978) apoyada por estudios posteriores (Elias \& Berk, 2002) acerca de la contribución del juego dramático al desarrollo de la auto-regulación, a todas las situaciones de juego. En este sentido podría pensarse que al emplear el lenguaje con esta función los niños están ensayando formas de regular la conducta propia y ajena durante las situaciones de juego.

Por otra parte, durante el juego dramático grupal y en rincones cerca de un tercio de las intervenciones lingüísticas de los niños tienen por objeto crear el mundo imaginario que sostiene el juego. En la textura de estos juegos, la representación se configura tanto a través de la dramatización de acciones como lingüísticamente. Asimismo, en estas situaciones los niños recurren en mayor medida a un lenguaje descontextualizado, en su intento de contribuir al progreso de la trama del juego. Como ha sido señalado por Pellegrini $(1985,1985 b)$ el juego dramático, a diferencia del juego de construcciones con objetos, al requerir el cambio de roles de la realidad a la fantasía, se sostiene a través de 
transformaciones verbales explícitas que evitan la ambigüedad en el juego. Los niños deben recurrir a formas en las que priman las referencias endofóricas -los elementos que se nombran, se definen y caracterizan dentro del texto y no por referencias al contexto de situación-. Asimismo requieren la definición de los objetos y de los personajes por medio de frases nominales complejas que incluyen modificadores directos e indirectos.

Los resultados del presente trabajo muestran que otras situaciones, al igual que el juego dramático, tales como la planificación y la explicación de las reglas en el momento previo al juego y la reconstrucción lingüística del juego en el intercambio posterior, dadas sus características comunicativas, conducen a los participantes a una descontextualización del "aquí y del ahora”. De ahí que demanden, por parte de los niños el empleo de un estilo de lenguaje explícito y un mayor nivel de integración de la información, estilo que, aún cuando se produce en la modalidad oral del lenguaje, es característico de la escritura. Es por ello que estas situaciones dan lugar a una intersección entre el juego y la alfabetización. El juego es, desde esta perspectiva, un generador de "zonas de desarrollo potencial” en las que se promueve la alfabetización.

Los resultados presentados coinciden con los hallazgos de Rosemberg (2008) y extienden en el plano lingüístico las observaciones de Bornstein, Haynes, Watson O’Reilly \& Painter (1996), de Tamis le Monda \& Bornstein (1993, 1994), de Tamis le Monda et al (1996) y de Sarlé (2000, 2005, 2006) que muestran que las intervenciones del adulto, cuando son contingentes con las propuestas infantiles, tornan más extensos y complejos los episodios de juego. En efecto, aún cuando los niños emplean lenguaje descontextualizado cuando interactúan con otros niños durante el juego dramático, lo hacen en mayor medida cuando interactúan con la maestra o bien durante el juego o bien en el momento previo para planificarlo o en el momento posterior para reconstruirlo lingüísticamente; es decir, en situaciones que les demandan la referencia a eventos lejanos en el espacio y en el tiempo.

De este modo, las situaciones de juego, de por sí contextos potentes para hablar y aprender, pueden volverse, en el marco de la interacción con un adulto, entornos en los que los niños desarrollan un esquema narrativo bien estructurado (Heath, 1982; 1982b) y un estilo de lenguaje descontextualizado (Pellegrini, 1985).

\section{Referencias}

Bondioli, A. (2001). The adult as a tutor in fostering children's symbolic play. En A. Gonku \& E. L. Klein (Eds.), Children in play, story and school (pp. 107- 131). New York, E.E.U.U: Guilford Press.

Bornstein, M. H., Haynes, O. M., Watson O’Reilly, A. \& Painter, K. M. (1996). Solitary and Collaborative Pretense Play in Early Childhood: Sources of Individual Variation in the Development of Representational Competence. Child Development, 67, 2910-2929.

Bruce, T. (1992). Children, adults and blockplay. En P. Gura \& T. Bruce (Eds.), Exploring Learning: young children and blockplay. London, England: Paul Chapman Publishing.

Bruner, J. (1986). El habla del niño. Barcelona, España: Paidós.

Chafe, W. (1985). Differences between speaking and writing. En D. Olson \& N. Torrance (Eds.), Literacy, language and learning. Norwood: Ablex.

Elias, C. L. \& Berk, L. E. (2002). Self regulation in young children: Is there a role for sociodramatic play? Early Childhood Research Quarterly 17, 216-238.

Enz, B. \& Christie, J. (1997). Teacher play interaction styles: Effects on play behaviour and relationships with teacher training and experience. International Journal of Early Childhood Education, 2, 55-69.

Garrido, R. (2008). Juegos con reglas y números. En Patricia Sarlé (Comp.), Enseñar en clave de juego. Enlazando juegos y contenidos (pp. 103-133). Buenos Aires, Argentina: Novedades Educativas. 
Halliday, M. A. K. (1975). Learning how to mean: explorations in the development of language. London, Inglaterra: Knobel.

Hauer, S. G; Altamura, V. O \& Anisfeld, M. (2007, 29 Marzo- 1 Abril). Pretend Play and Early Language Development. A study of Brazilian Toddlers aged 16 to 28 months. Poster presentado en Society for Research in Child Development Biennial Meeting, Boston, Massachusetts, USA.

Heath, S. B. (1982). What no bedtime story means; Narrative skills at home and at school. Language in Society, 11, 49-76.

Heath, S. B. (1982b). Questioning at home and at school: a comparative study. En J. D Spindler (Ed.), Doing the ethnography of schooling: Educational anthropology in action. New York, E.E.U.U: Holt, Rinehart y Winston.

Manrique, M. S. (2009). Conversación y desempeño lingüístico en la infancia. Un estudio de la interacción verbal en el Jardín de Infantes con población urbano - marginal. Tesis doctoral inédita. Facultad de Filosofía y Letras. Universidad de Buenos Aires. U. B. A.

Morrow, L. M. \& Schickedanz, J. A. (2006). The relationships between Sociodramatic Play and Literacy Development. En Dickinson K. D y Neuman, S. B (Eds.), Handbook of Early Literacy Research. Vol 2 (pp. 269-280). N.J, E.E.U.U: Guilford Press.

Nelson, K. (1996). Language in cognitive development. Cambridge, England: Cambridge University Press.

Pellegrini, A. D. (1982). The construction of cohesive text by preschoolers in two play contexts. Discourse processes, 5, 101 -108.

Pellegrini, A. D. (1984). The effects of Classroom Ecology on Preeschooler's Functional Uses of Language. En A. Pellegrini \& T. Yawkey (Eds.), The development of oral and written language in social contexts (pp. 129- 141). Norwood, N. J: Ablex.

Pellegrini, A. D. (1985). Relations between preschool children's symbolic play and literate behavior. En L. Galda \& A. Pellegrini (Eds.), Play language and stories (pp. 79- 97). Norwood, N.J: Ablex.

Pellegrini, A. D. (1985b). Play Centers and the Production of Imaginative Language. Discourse Processes 9, 115-125.

Piaget, J. (1962) Play, dreams and imitation in childhood. New York, E.E.U.U: Norton.

Rivière, A. (2003). El juego simbolico en ninos ciegos. En J. M. Ruiz-Vargas \& M. Belichon (Comp.), Angel Rivère. Obras Escogidas. Vol II. Lenguaje, simbolización y alteraciones del desarrollo (pp. 173-192). España: Ed. Médica Panamericana.

Rosemberg, C. R. (2008). El lenguaje y el juego en la educación infantil. En Patricia Sarlé (Comp.), Enseñar en clave de juego enlazando juegos y contenidos (pp. 61-75). Buenos Aires, Argentina: Novedades Educativas.

Sarlé, P. (2000). El juego dramático, la educación infantil y el aprendizaje escolar. Psykhè, Vol 9 $N^{\circ} 2,41-53$.

Sarlé, P. (2005). El juego dramático en la escuela infantil. Revista Novedades Educativas, Edición $171,23-27$.

Sarlé, P. (2006). Enseñar el juego y jugar la enseñanza. Buenos Aires, Argentina: Paidós.

Tamis le Monda C. S. \& Bornstein M. H., (1993). Play and its relations to other mental functions in the child. En M. H. Bornstein \& A. O’Reilly (Eds.), New directions for child development: The role of play in the development of thought, Vol. 59 (pp.17-27). San Francisco, CA, E.E.U.U: Jossey-Bass. 
Tamis le Monda C. S. \& Bornstein M. H. (1994). Specificity in mother-toddler language-play relations across the second year. Developmental Psychology, 30, 283-292.

Tamis le Monda, C. S., Bornstein, M. H., Baumwell, L \& Damast, A. M. (1996). Responsive parenting in the second year: specific influences on children's language and play. Early Development and Parenting, 5, 173-183.

Vygotski, L. (1978). Mind in society: The development of higher mental processes. En M.Cole, V. John- Steiner, S. Scribner, \& E. Souberman (Eds. \& Trans.). Cambridge, Inglaterra, MA: Harvard University Press.

Vygotski, L. (1988). El juego en el desarrollo del niño. En Vygotski, L. (1988). El desarrollo de los procesos psicológicos superiores. Barcelona, España: Ed. Crítica. 